\title{
Heartbeat: Virtual histopathology after myocardial infarction
}

Inflammation plays a key role in outcomes after myocardial infarction yet we have few tools to directly visualise the cellular inflammatory response in clinical practice. In this issue of Heart, Stirrat and colleagues $^{1}$ performed repeated MRI studies in 31 patients after myocardial infarction using ultra-small-super-paramagnetic particles of iron oxide (USPIO) to visualise macrophages within the myocardium. In combination with other MRI imaging sequences, this approach provides a virtual histopathologic view of the myocardium. In the infarcted myocardial segment, USPIO uptake peaked at 2-3 days and resolved by 10-16 days whereas myocardial oedema peaked later (day 3 to 9) and persisted for over 3 months. (figure 1) As the authors suggest: "This imaging technique holds promise as a non-invasive method of assessing and monitoring myocardial cellular inflammation with potential application to diagnosis, risk stratification and assessment of novel anti-inflammatory therapeutic interventions."

In an editorial, Yilmaz ${ }^{2}$ comments that this data support the concept of two distinct phases in recovery after myocardial infarction: "a first inflammatory phase (until day $\sim 4$ following acute MI) that is characterised by the accumulation of proinflammatory monocytes (CD16-) that primarily remove necrotic cells and debris and a second resolution phase (following day $\sim 4$ after acute $\mathrm{MI})$ in which reparative monocytes/macrophages $(\mathrm{CD} 16+)$ predominate and orchestrate myocardial tissue repair." Further, he suggests that USPIO-enhanced MRI may allow noninvasive detection and monitoring of myocardial inflammation and differentiation of a recent (within 2 weeks) from more remote myocardial infarction. Future applications of this imaging approach might include other causes of myocardial inflammation-for example transplant rejection, myocarditis, or sarcoidosis.

A subset of patients with bicuspid aortic valve (BAV) disease have an associated aortopathy that primarily affects the aortic sinuses and ascending aorta. This association has raised the concern that BAV disease might also be associated with a higher prevalence

Correspondence to Professor Catherine M Otto, Division of Cardiology, University of Washington, Seattle, Washington, USA; cmotto@uw.edu

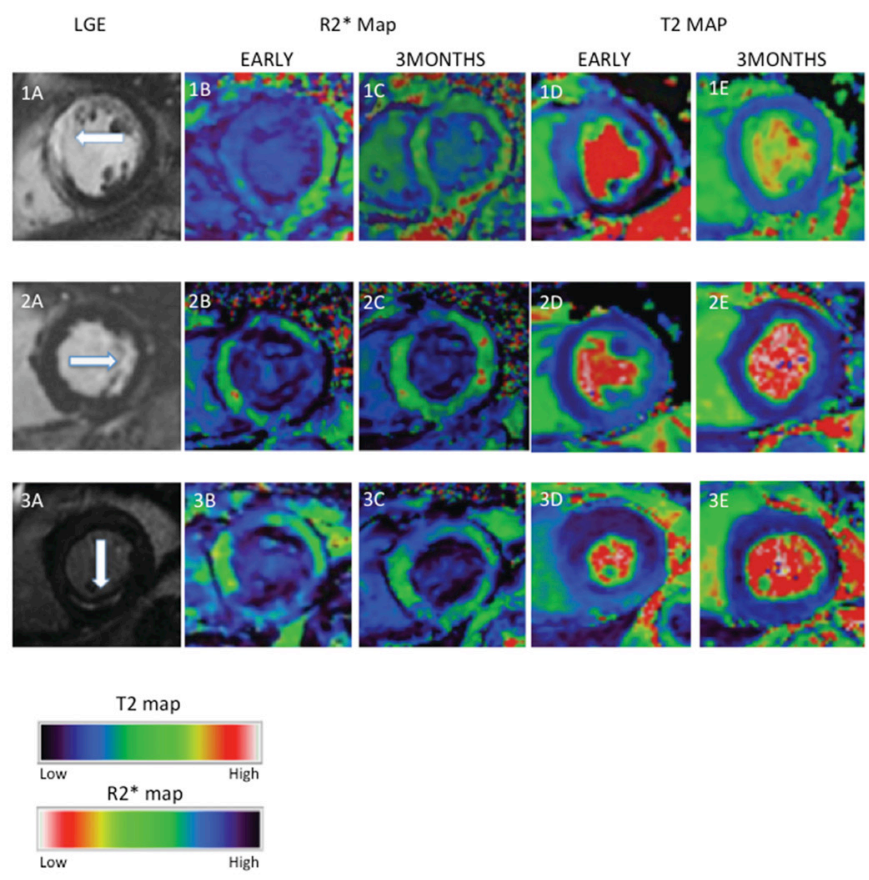

Figure 1 Examples of myocardial oedema and USPIO enhancement in the infarct zone after MI. Three examples of MI (1-anteroseptal, 2-lateral and 3-inferior) illustrating LGE, USPIO enhancement (R2* map) and oedema (T2 map) at early (up to 10 days) and late (3 months) time points. Early inflammation and oedema seen on R2* (dark region) and T2 maps (light region), respectively, have improved or resolved by 3 months. LGE, late gadolinium enhancement; $\mathrm{MI}$, myocardial infarction; USPIO, ultrasmall superparamagnetic particles of iron oxide.

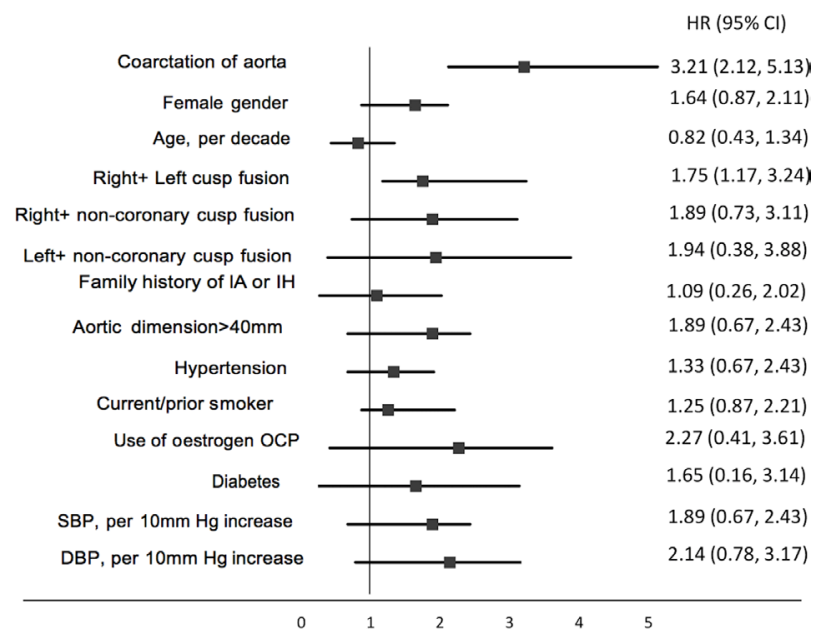

Figure 2 Predictors of intracranial aneurysm (IA). DBP, diastolic blood pressure; IIH, intracranial haemorrhage; OCP, contraceptive pill/oral contraceptives; SBP, systolic blood pressure.

of other vascular malformations, particularly intracranial aneurysm (IA). In a retrospective review of $678 \mathrm{BAV}$ patients who underwent brain magnetic resonance angiography, Egbe ${ }^{3}$ and colleagues observed an IA in $7.7 \%$ of patients overall, with a higher prevalence in those with BAV plus aortic coarctation $(20 / 154,12.9 \%)$ compared than in those with an isolated BAV (32/524, 5.7\%) (figure 2). The risk of IA was not associated with age, there were no new cases detected over 7 years of follow-up, and the risk of rupture in those with an IA was 4\% over 11 years of follow-up. 

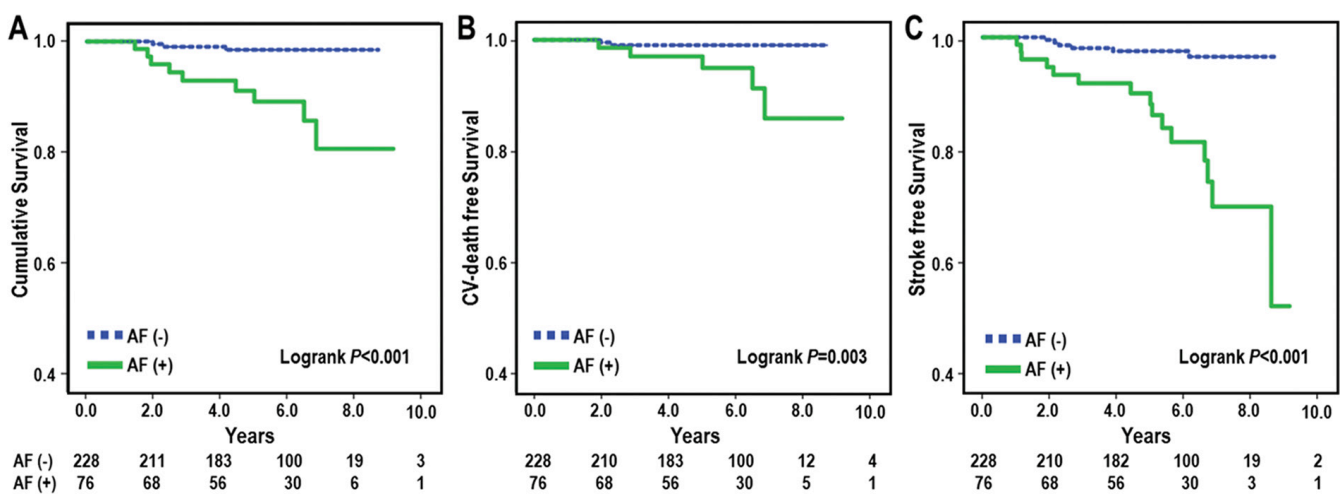

Figure 3 Kaplan-Meier survival curve of the overall survival (A), cardiovascular (CV) death-free survival (B) and stroke-free survival (C) according to atrial fibrillation (AF). Patients with AF had a significantly lower cumulative survival-free rate of total deaths $(p<0.001), C V$ death $(p=0.003)$ and strokes $(p<0.001)$ than those without AF.

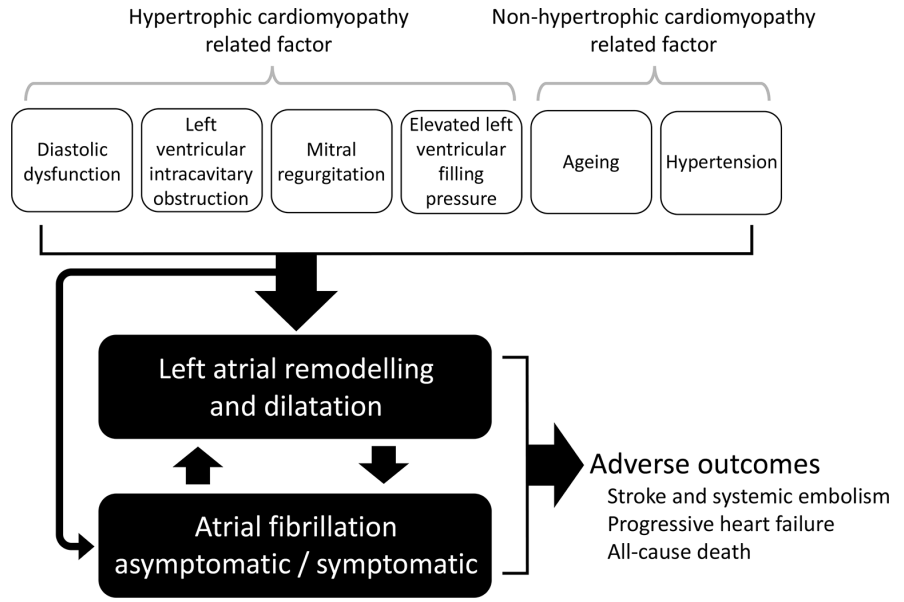

Figure 4 Left atrial remodelling and dilatation, development of atrial fibrillation and adverse outcome in patients with hypertrophic cardiomyopathy.

It is likely that this retrospective study overestimates the risk of IA because brain magnetic resonance angiography was performed for clinical indications, which is not routinely done for screening of BAV patients at most institutions and is not recommended in current guidelines. There also was a very high prevalence of associated aortic coarctation, suggesting these patients may differ from the more typical patient with isolated BAV disease. Even so, Girdauskas $^{4}$ and the study authors suggest that baseline brain magnetic resonance angiography may be reasonable in BAV patients, particularly women and those with an aortic coarctation. Girdauskas also notes that the prevalence of the reverse association is not clear; in patients with an IA, clinical series report a BAV prevalence ranging from $0.6 \%$ to $10 \%$. He concludes: "Given this marked heterogeneity of BAV disease, specific BAV phenotypes might be hypothetically associated with a different potential for IA formation. Due to quite infrequent coexistence between BAV and IA, only a large population-based longitudinal study comparing IA prevalence in (bicuspid versus tricuspid aortic valve) entities, with and without concomitant aortopathy, could potentially provide a reliable answer to this intriguing question."

There is limited data on long-term outcomes in patients with apical hypertrophic cardiomyopathy (ApHCM). In a consecutive series of $306 \mathrm{ApHCM}$ patients, the $25 \%$ of patients with atrial fibrillation (AF), compared with those who remained in sinus rhythm, had a higher incidence of all cause $(11.7 \%$ vs $1.3 \%)$ and cardiovascular $(6.5 \%$ vs $0.9 \%$ ) death over an average follow-up of 5.5 years (figure 3$).^{5}$ As might be expected, the incidence of stroke was higher with AF compared with sinus rhythm $(19.5 \%$ vs $2.6 \%)$ even when adjusted for age and sex. However, AF was detected before stroke in only $53 \%(8 / 15)$ of patients. In addition, $75 \%(6 / 8)$ of patients with known AF were on anticoagulation at the time of stroke.

Minami and Hagiwara ${ }^{6}$ point out that outcomes in this cohort of ApHCM patients are similar to those reported for other forms of HCM. They emphasise that we need to address management of AF, not just prevention of sudden cardiac death, in these patients given the association of $\mathrm{AF}$ with adverse outcomes. Several factors contribute to left atrial enlargement and atrial fibrillation in
HCM patients (figure 4). They conclude: "Finally, to improve the prognosis of patients with apical and non-apical HCM, further optimisation of risk stratification, screening methods and management strategies for $\mathrm{AF}$ remains a clinical challenge."

See if you can figure out the Image Challenge in this issue. ${ }^{7}$ Difficult placement of catheters for an electrophysiology procedure led to chest computed tomographic imaging with unexpected findings in a 72 year old woman with palpitations.

\section{Competing interests None declared.}

Provenance and peer review Commissioned; internally peer reviewed.

(C) Article author(s) (or their employer(s) unless otherwise stated in the text of the article) 2017. All rights reserved. No commercial use is permitted unless otherwise expressly granted.

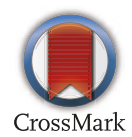

To cite Otto CM. Heart 2017:103:1473-1474.

Heart 2017:103:1473-1474.

doi:10.1136/heartjnl-2017-312355

\section{REFERENCES}

1 Stirrat CG, Alam SR, MacGillivray TJ, et al. Ferumoxytolenhanced magnetic resonance imaging assessing inflammation after myocardial infarction. Heart 2017;103:1528-35.

2 Yilmaz A. Visualising inflammation after myocardial infarction with the use of iron oxide nanoparticles. Heart 2017;103:1479-80.

3 Egbe AC, Padang R, Brown RD, et al. Prevalence and predictors of intracranial aneurysms in patients with bicuspid aortic valve. Heart 2017;103:1508-14.

4 Girdauskas E. Bicuspid aortic valves and intracranial aneurysms: more than an incidental coexistence? Heart 2017:103:1477-8.

5 Lee S-E, Park J-K, Uhm J-S, et al. Impact of atrial fibrillation on the clinical course of apical hypertrophic cardiomyopathy. Heart 2017;103:1496-501.

6 Minami Y, Hagiwara N. Enlarged left atrium, atrial fibrillation, and adverse outcome in hypertrophic cardiomyopathy. Heart 2017:103:1475-6.

7 Martin CA, Gajendragadkar PR, Agarwal S. Palpitations in a 72 year old woman. Heart 2017;103:1554-5. 